\title{
Self-Assessment Program
}

\section{Blood conservation and the management of periop- erative blood transfusions in a patient undergoing major vascular surgery: a Self-Assessment Program}

\begin{abstract}
Members of the CAS and subscribers to the CJA are first invited to read the introduction that follows and the articles cited in the bibliography to prepare for the Self-Assessment Program. The reader should then go to the Journal's website (www.cja-jca.org) for the Problem Based Learning session. Completion of the Self-Assessment Program will entitle subscribers to claim ten hours of Continuing Professional Development (CPD) under section 3 of CPD options, for a total of 20 Maintenance of Certification credits (note that section 3 hours are not limited to a maximum number of credits per five-year period). There is no requirement to succeed: the goal of participating is to define potential areas for improvement.
\end{abstract}

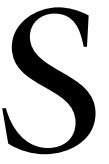

INCE the discovery of human blood groups by Landsteiner in 1900 major advances in transfusion medicine have occurred, making current blood supply safer and safer. However, despite the efforts made to reduce the risks associated with blood transfusion, the risk will never be zero. Non infectious hazards of transfusion as well as misidentification of crossmatch samples or recipients with possible fatal consequences are true and exist. In addition, with the discovery of new viruses with an unknown risk of pathogenicity and the possible transmission of the agent responsible for Creutzfeld-Jacob disease, blood components will probably never be completely safe. By the mid-80s, it had become obvious, progressively, that transfusion of blood and blood products should be reduced and kept to a minimum.

Blood conservation is a global concept including all possible strategies aimed at reducing patients' exposure to allogeneic blood products. Numerous techniques have been developed, which vary in efficacy, timing, mechanisms of action, and in the level of knowledge required to use them. They have costs and also carry some risks. The selection of the most appropriate technique(s) in a given clinical setting remains a difficult task. The goal of the Supplement "Perioperative blood conservation strategies: an update for clinicians" published in 2004 in the Canadian Journal of Anesthesia was to provide clinicians pertinent information on most, but not all, avail- able techniques. These will assist them in building strategies best adapted to the patients under their care on a day-to-day basis.

Information spanning the entire process must be taken into account, from the moment an operative decision is considered until the patient returns home in a normal functional status. With this approach, the concept of blood conservation rests on three pillars: increasing the red blood cell mass of the patient, decreasing perioperative blood losses, and optimizing blood transfusion practice.

The need to increase red blood cell (RBC) production as part of a general strategy to reduce blood transfusion and a better understanding of the physiopathology of different forms of anemia have resulted in an increased interest in the use of iron. Dr. Beris reviewed the different routes of iron administration and discussed indications for oral $p s$ iv iron supplementation. ${ }^{1}$ Despite approval for the perisurgical use of erythropoietin (EPO) in western countries in the mid-90s, acceptance for its utilization as an alternative to blood transfusion remains slow. Dr. Goodnough developed arguments that could improve cost-effectiveness of EPO therapy, with or without autologous blood procurement, in the perioperative setting. ${ }^{2}$

Keeping blood loss to a minimum is the first step to avoid or to reduce allogeneic blood exposure. Dr. Ozier discussed some of the non-pharmacological approaches that can be easily integrated into daily anesthetic care. ${ }^{3}$ 
Among them, prevention of intraoperatively acquired coagulopathies will truly benefit anyone and should be considered one of the first step of blood conservation strategies. Dr. Levy developed current and future pharmacological approaches aimed at attenuating activation of the hemostatic system, in order to decrease coagulopathy and the potential need for blood transfusion, and at producing specific hemostatic activation in the bleeding patient. ${ }^{4}$ In modern surgical practice, RBC salvage represents a key component of any blood conservation strategy. Dr. Rubens discussed the principles and mechanics of cell salvage (unprocessed and processed) and reviewed the safety and the efficacy of the different techniques that can be used in reducing the need for allogeneic blood administration. ${ }^{5}$ Artificial oxygen carriers, including modified hemoglobin solutions and perfluorocarbon emulsions have been developed in an attempt to replace blood transfusion. Dr. Spahn et al. described the currently evaluated compounds, summarized their efficacy and discussed their side-effects. ${ }^{6}$ Clearly, the potential use of these products will require education of healthcare professionals to familiarize them with these new concepts and physiology.

The third axis of blood conservation is the adequate use of blood products. The presence of significant variation in blood transfusion rates implies that the best practice has yet to be identified and that indications for transfusion are not consistent among providers. This is specially true during massive transfusion, as outlined by Hardy et al. Massive bleeding and transfusion will often result in hemostatic dysfunction, the extent of which will vary according to the clinical circumstances (hypothermia, bleeding during elective surgical bleeding, trauma, etc.). ${ }^{7}$ Treatment should take into account these circumstances and the results of diagnostic laboratory testing.

A better knowledge of the physiological responses during acute and chronic anemia, of the clinical factors that can limit adequate tissue oxygenation during anemia and of the potential benefits of blood transfusion will allow the clinician to better define transfusion criteria for each patient. Dr. Henny reviewed the physiology of anemia, emphazising situations where acute normovolemic anemia is superimposed upon chronic anemia. ${ }^{8}$ Anemia is a frequent finding in the critically ill patient. Dr. Vincent et al. discussed the common causes and possible approaches to prevent and to treat anemia in this high risk patient population. ${ }^{9}$ Patients undergoing surgery are often elderly and have comorbidities. Anemia may impede the ability of these patients to recover optimal postoperative functional status. Dr. Carson et al. reviewed the basis for an effect of anemia on postoperative recovery and the literature describing the relationship between anemia and func- tion. ${ }^{10}$ Benefits of blood transfusion refer to the capacity of transfusion to correct the risks associated with anemia and possibly provide additional benefits. Dr. Hébert et al. examined the efficacy of RBC transfusion through a systematic review of the literature comparing restrictive to liberal transfusion strategies. ${ }^{11}$

Despite the wealth of information provided by the experts, it is clear that there is no one single blood conservation strategy that will benefit all surgical patients. In addition, we realize fully that not all techniques may be available in a given institution. This must be kept in mind when answering the questions included in the Self-Assessment Program. The reader should provide the best possible answer, understanding that some of the other options provided may also be, at least in part, appropriate management strategies.

Jean-François Hardy MD*

Philippe Van der Linden MD $\dagger$

University of Montréal, ${ }^{*}$ Montréal, Canada

CHU Brugmann, $\dagger$ Brussels, Belgium

\section{Bibliography}

1 Beris $P$. The use of iron to increase red cell mass. Can J Anesth 2003; 50(6 Suppl): S3-9.

2 Goodnough LT. The use of erythropoietin to increase red cell mass. Can J Anesth 2003; 50(6 Suppl): S10-8.

3 Ozier $\Upsilon$, Lentschener C. Non-pharmacological approaches to decrease surgical blood loss. Can J Anesth 2003; 50(6 Suppl): S19-25.

4 Levy JH. Novel pharmacologic approaches to reduce bleeding. Can J Anesth 2003; 50(6 Suppl): S26-30.

5 Rubens FD, Boodbwani M, Lavalee G, Mesana T. Perioperative red blood cell salvage. Can J Anesth 2003; 50(6 Suppl): S31-40.

6 Spabn DR, Kocian R. The place of artificial oxygen carriers in reducing allogeneic blood transfusions and augmenting tissue oxygenation. Can J Anesth 2003; 50(6 Suppl): S41-7.

7 Hardy JF, De Moerloose P, Samama M. Massive transfusion and coagulopathy: pathophysiology and implications for clinical management. Can J Anesth 2004; 51: 293-310.

8 Henny CP, Trouwborst A. Physiology of acute vs physiology of acute vs chronic anemia. Can J Anesth 2003; 50(6 Suppl): S48-52.

9 Vincent JL, Sakr $\Upsilon$, Creteur J. Anemia in the intensive care unit. Can J Anesth 2003; 50(6 Suppl): S53-9.

10 Carson JL, Terrin ML, Jay M. Anemia and postoperative rehabilitation. Can J Anesth 2003; 50(6 Suppl): S60-4.

11 Hebert PC, McDonald BJ, Tinmouth A. Overview of transfusion practices in perioperative and critical care. Can J Anesth 2003; 50(6 Suppl): S65-75. 